\title{
Trabajo conjunto familia y docente, el caso de un padre con su hijo autista: Una experiencia de investigación acción
}

\author{
Teacher and Family Working Together, the Case of a Father and his Autistic Son: \\ An Action Research Experience
}

\author{
María del Rocío Rodríguez-Roblero' \\ Escuela Nuestra Señora de Lourdes \\ Heredia, Costa Rica \\ pochy26@gmail.com
}

Recibido 3 de noviembre de 2013 • Corregido 11 de octubre de 2014 • Aceptado 22 de noviembre de 2014

\begin{abstract}
Resumen. Este artículo explica el proceso de investigación acción vivido durante un año con una familia, muestra el trabajo realizado con el padre, la docente y un niño con autismo. Surge a partir de la pregunta: ¿Cómo trabajar en conjunto padre y docente para incidir positivamente en el proceso educativo humanizante de un niño con autismo? Se presentan las reflexiones que surgieron a partir deesta experiencia, la incertidumbre y las angustias propias de una docente que no sabe de dónde partir para trabajar con la familia. La investigación acción fue el medio para trabajar y encontrar espacios para compartir la búsqueda de una respuesta. Se utilizó el juego como instrumento mediador de estos espacios entre docente, padre y niño. A partir del análisis de las observaciones, en cada una de las sesiones nacen los aprendizajes en conjunto. Se evidenció la importancia de la participación de la familia en los entornos educativos no como simples receptores, si no como protagonistas del proceso.
\end{abstract}

Palabras claves. Acompañamiento, familia, resiliencia, transformación, investigación acción, autismo.

Abstract. This paper describes the action research process experienced with a family over one year and shows the work done by the father, the teacher and an autistic child. The question proposed was: How do parents and the teacher work together to have a positive impact on the humanizing educational process of an autistic child? The paper presents the reflections emerging from this experience, as well as the uncertainty and anxiety of a teacher who does not know where to start working with the family. The action research model was the means to work and find spaces to jointly search for an answer. Playing was the instrument used to mediate in these spaces between the teacher, the parent and the child. The analysis of the observations shows that joint learning is brought about in each session. This research project evidenced the importance of family involvement in educational environments, not merely as recipients but as participants in the process.

Keywords. Accompaniment, family, resilience, transformation, action research, autism.

\footnotetext{
${ }^{1}$ Licenciada en Educación Preescolar de la Universidad de Costa Rica. Ha realizado cursos de actualización profesional en instituciones como la Universidad Nacional y Universidad de Costa Rica. Actualmente trabaja en el Centro Bilingüe Nuestra Señora de Lourdes de Heredia, donde tiene a cargo el grupo de Maternal II y es egresada de la Maestría Profesional en Pedagogía con Énfasis en Desarrollo y Atención Integral de la Primera Infancia de la Universidad Nacional de Costa Rica.
} 
doi: http://dx.doi.org/10.15359/ree.19-1.16

URL: http://www.una.ac.cr/educare

CORREO: educare@una.cr

Todo cambio en la vida trae nuevas aventuras, la que se presenta en este artículo es una de ellas y comenzó ya hace más de un año. Esta investigación surge en el marco de la Maestría en Pedagogía con énfasis en Desarrollo y Atención Integral de la Primera Infancia e inicia en el momento en el que como docente me pregunto qué hacer y cómo actuar para responder a las necesidades de un niño con autismo que se integra a mi clase de educación preescolar. El año pasado mi quehacer supuso una situación particular, nunca antes había integrado un niño con alguna necesidad educativa en mi clase. Lo más difícil no fue trabajar con el niño sino cómo integrar a la familia en el proceso educativo de su hijo, me pregunto constantemente cómo trabajar juntos para aportarle al niño el máximo de apoyo. Se trabajó con un padre que no vive con su hijo; pero busca como ir entendiendo el autismo y acercase a su hijo.

Llena de dudas e incertidumbre no sabía por dónde empezar. Me cuestiono acerca de mi función como docente, ¿cuál es su esencia?, ¿cuáles sus límites?, ¿cuáles sus potenciales?, ¿qué es lo que debo hacer? Estas preguntas me llevan a hacer un alto en el camino para dar cabida a la sistematización, la reflexión, la acción. Prueba-error, aprendizaje-desaprendizaje son las herramientas que por un año me acompañan sistemática y sistémicamente en aras de cambiar y crecer como profesional (Katz, 2005).

Es evidente, entonces, que el paradigma desde el cual se articula este proceso investigativo es el naturalista. Como preguntas generadoras iniciales me planteo: ¿Cómo influirnos y ayudarnos mutuamente familia y docente? ¿Cómo incidir en el proceso educativo del niño a partir de nuestro trabajo conjunto?

Busco comprender el papel del padre, romper algunos esquemas o prejuicios que como docente suelo tener acerca de lo que debe hacer un padre, su lugar en el proceso educativo, su lugar frente al aprendizaje y desarrollo del niño, su lugar como soporte emocional y de cuidado. En tanto vínculos, aspiraciones y papeles por asumir, el proceso de investigación me lleva a develar los valores que influyen en el proceso, mis valores como docente, mis creencias, mi manera de pensar acerca del papel del padre, en la educación y el apoyo a sus hijos. Desde la perspectiva axiológica, acepto que nuestros espacios de intercambio son siempre campos de sentido que se interconectan y trascienden las acciones de un momento histórico. Cada encuentro con el padre es un encuentro histórico, social y técnico (Dobles, Zuñiga y García, 1998).

El ejercicio de reflexión durante el proceso siempre estuvo mediado por la humildad necesaria para aceptar que nos equivocamos y la resiliencia para ver en la adversidad una oportunidad (Kotliarenco, Cáceres y Álvarez, 2011). En consecuencia, asumo que la construcción de conocimientos surge y se fortalece en las relaciones humanas, que el error es condición imperativa del proceso y que los saberes circulan entre todos los participantes. El saber acerca de la paternidad y del rol docente reside en cada persona que asume este papel, este saber nos determina, a la vez que lo determinamos y modificamos constantemente en el diario vivir. 
Somos parte y productores de este saber conjugado con los otros, como sujetos activos que nos permiten poco a poco articular y crear, en aras de comprender para intervenir en este proceso de aprendizaje de la manera más asertiva posible (Morin, 2003).

La perspectiva epistemológica que caracteriza este proceso de investigación es dialéctica e interactiva porque busca la participación de todos de manera activa. Según Ferrer (2012), bucear en la educación es tan complejo como profundo. Cuando los padres desean asomarse en este gran mundo, se encuentran perdidos. La educación de los hijos comienza pronto, mucho más temprano de lo que nos imaginamos. Por lo que el papel de los padres y madres y, en nuestro caso, de la docente que acompaña es fundamental. Aprendemos juntos en interacción.

De modo que desde la mirada ontológica, la realidad a la que nos acercamos es la nuestra, compartida, padre, docente y niño. Cada uno con supuestos asumidos y roles a cargo, algunos conscientes y otros no tanto. Todos coincidiendo en un punto común: la vida del niño y los múltiples intereses o deseos que develaremos con el diálogo y la convivencia. Un mosaico de realidades articuladas, compartiendo un tiempo y un espacio (Dobles et al., 1998).

En suma, se trata de un proceso de investigación abierto a lo desconocido, al aprendizaje en el trabajo con el niño y su padre en el diario vivir. Para mí es fundamental tener una visión amplia y traspasar las fronteras del saber y del quehacer, en especial respecto a cómo intervenir ante las etiquetas, que en algunas ocasiones le asignamos a nuestros niños, ¿cómo no permitir que nos atrapen y comprender al ser humano tras ellos?, ¿cómo ser flexible y aprovechar las oportunidades para trabajar de maneras diversas? Con base en los argumentos propuestos emerge la siguiente pregunta de investigación: ¿Cómo trabajar en conjunto padre y docente para incidir positivamente en el proceso educativo humanizante de un niño -con autismo-? La información que se comparte, se articula desde la investigación acción participativa, la que incide y se desarrolla sobre la práctica, realizada por los prácticos y para estos. Chaves (2007) destaca que es un tipo de investigación que realiza el personal docente en sus salones, para analizar una problemática y buscar soluciones mediante un plan de acción. Latorre (2004), por su parte, menciona que es un proceso de carácter cíclico. Estos ciclos van generando transformaciones tanto en las personas como en el proceso en sí, en busca de soluciones y sobretodo de reflexión acerca de lo que se está haciendo. Esos cambios llevan a una transformación por parte de todos los participantes.

\section{Ruta metodológica, camino hacia la transformación}

En la investigación en el trabajo con el padre se realizaron encuentros, pequeños espacios donde los participantes compartíamos, estos encuentros se dividieron en tres momentos.

El primero tiene como eje el ensayo y error en el trabajo, constituye el espacio-tiempo en el cual nos conocemos, acercamos, probamos maneras diversas de interactuar cada quien en un 
doi: http://dx.doi.org/10.15359/ree.19-1.16

URL: http://www.una.ac.cr/educare

CORREO: educare@una.cr

rol desarticulado del otro, cada quien cumpliendo objetivos didácticos, de crianza, entre otros. Posteriormente esto se va desvaneciendo para dar lugar al segundo momento, que se caracteriza por dos condiciones principales, el paso del tiempo y la profundidad de la comprensión de la subjetividad del otro en los intercambios. Esto nos lleva a cambios y ajustes en el trabajo conjunto. Un tercer momento crece exponencialmente, es el relacionado con la reflexión y análisis acerca del proceso realizado, del cual emerge la ruptura epistemológica en este proceso.

Parto de un tipo de investigación participativa, que surge a partir de un problema y preguntas acerca de mi práctica laboral cotidiana: ¿Qué es lo que debo hacer? ¿Soy guía, acompañante, apoyo, orientación? ¿Cómo se debe trabajar con las familias? ¿Cómo influirnos y ayudarnos mutuamente familia y docente? ¿Cómo incidir en el proceso educativo del niño a partir de nuestro trabajo conjunto?

El intercambio de aprendizajes comienza con una entrevista inicial, para conocer la disposición del padre a realizar el trabajo conjunto. A partir de la entrevista, acordamos reunirnos dos veces por semana, en la escuela, antes de empezar la clase, de 6:30 am a 7:00 am. Esto para responder a las necesidades y características del estilo de vida del padre, adaptarme a sus horarios y espacios contextuales. Trabajamos en total 12 sesiones. En términos del proceso de transformación las clasifiqué en tres tipos:

1. Actividades para la comprensión del trabajo. La denominé de esta manera, porque tanto para el padre como para mí estas primeras sesiones fueron de comprensión mutua; buscábamos entender nuestra manera de trabajar y de ver de qué manera se ayudaba al niño.

2. Actividades de aprendizaje y comprensión de su hijo. Su nombre alude a que en estas sesiones el trabajo más importante fue buscar comprender más allá de la actividad, es decir, lograr la comprensión y el reconocimiento de su propio hijo. En todo momento se aprendió por parte de todos los participantes.

3. Espacios de disfrute padre-hijo-docente. Esta parte final de las sesiones de trabajo recibe este nombre porque se dio un cambio de parte de los participantes y se lograron espacios de disfrute de padre-hijo-docente.

Cabe mencionar que las primeras actividades fueron diseñadas para aplicar en cada sesión, considerando las necesidades del niño y tomando en cuenta las experiencias de la sesión anterior, siempre se valoró lo que funcionó y lo que no antes de plantear la nueva actividad. Como docente, al sistematizar lo ocurrido en estas sesiones con crónicas diarias de cada uno de los momentos, remiro mi quehacer cotidiano con el niño y con el padre desde otra perspectiva, para ser más crítica acerca de mi papel pedagógico, para no repetir lo que es factor de debilidad y desgaste (Jara, 1994). 
Pretendía un cambio en mi práctica educativa, buscaba como involucrar a la familia en la tarea pedagógica; pero requería tomar distancia y mirarla desde otra perspectiva para poder renovarla (Chaves, 2007). Buscaba caminos de transformación y encontré en la investigación acción esa oportunidad para hacerlo y, mediante la reflexión sistemática, me llevó a la acción (Latorre, 2004).

El "diagnóstico de autismo" es un elemento que nos influyó, como elemento interruptor que capta la mirada, que desdibuja al niño frente a la etiqueta. Nos generó ansiedad e impotencia por desconocimiento. Sin embargo, a medida que interactuamos aparece como una característica o condición, y no como un impedimento en el proceso. El mayor reto fue impedir que el autismo se volviese un cuarto participante cegador, para ver más allá: de lo que se trataba era de ver al niño y no su etiqueta.

Mis objetivos pedagógicos en estas sesiones fueron abrir un espacio diferente de interacción organizado desde la acción lúdica, donde las manifestaciones de afecto y la comunicación se abriesen camino poco a poco. Elegí iniciar guiando el proceso, aun así me cuestionaba permanentemente: ¿Estaré siendo una guía? ¿Estaré conduciendo el proceso de la mejor manera? ¿Cuánto estoy interviniendo? ¿Cómo lo hago? ¿Cuál debe ser mi participación? Inclusive, ¿cuál es el rumbo que este proceso debe tomar?

Estaba clara en que no quería ser yo quien dirigiera los juegos; padre e hijo debían encontrar su propio camino en la interacción. Me sentía temerosa con el padre, no sabía qué hacer con él, como modificar su rol. Las primeras dos semanas propuse que jugaran con plasticina y con juguetes de ensartes y de enhebrar; busqué juegos sencillos para que el padre se integrara y eran el medio para incentivar el intercambio entre padre, niño, docente. No tuve buenos resultados, las interacciones - desde mi perspectiva- no se daban, porque el padre no participaba. Al analizar estas sensaciones descubrí que estaba tratando, sin darme cuenta, de que padre e hijo igualaran mi ritmo de aprendizaje, en lugar de autorregularme al de ellos. Coincido con Chaves (2007) en que la investigadora no es neutral en las decisiones que adopta, sino que se proyecta en valores y planteamientos éticos. Mi responsabilidad es, entonces, poco a poco concientizarme de mis prejuicios, intencionalidades y creencias, de manera que no se constituyan en elementos interruptores de proceso.

Para mí, era difícil dejar de dar indicaciones, quería que el padre tomara un papel participativo. ¿Qué significa participativo para mí? ¿Cómo pasar de lo que yo quiero o decido a un lugar de acompañante y promotora de escucha? Era importante buscar alternativas, en particular para analizar mi actuar. Reflexiono que interactuar con este padre y este niño es más que planear actividades y adaptarlas a nuestra realidad compartida como sujetos activos.

Para el niño, su padre es alguien con quien se identifica, es alguien que le apoya en su evolución social (Dolto, 2000). Este padre, con sus aciertos y limitaciones, cumple este importante papel. ¿Me corresponde calificar como positivas o negativas sus intervenciones? 
doi: http://dx.doi.org/10.15359/ree.19-1.16

URL: http://www.una.ac.cr/educare

CORREO: educare@una.cr

¿Debo mostrarle cómo acercarse a su hijo? ¿Está alejado de su hijo o sobrecogido por la etiqueta que otros profesionales le impusieron y en torno a la cual hemos articulado las intervenciones?

Tengo la posibilidad de respetar el estilo de vinculación existente entre padre e hijo, desarrollando una mirada inclusiva, cercana y no prejuiciosa del papel de las personas adultas de la familia. Este es mi reto, mi oportunidad de mejora comienza a tomar forma.

Debo prestar atención a tres aspectos: el vínculo, qué sabe el padre del autismo y mis conocimientos reales de las necesidades de esta familia. Me pregunto ¿qué tanto conozco al padre y al niño, su realidad y contextos? Es desde el conocimiento de dicha realidad, que la intervención se transforma en su intencionalidad e impacto. Entonces, me doy cuenta de que no solamente las sesiones o lo que se pueda hacer en el aula es importante; debo conocer a esta familia, sus necesidades afectivas, sociales, educativas, de información, en aras de enriquecer mi comprensión del proceso de desarrollo del niño. Se trata de ir más allá de mi perspectiva, mi visión de las cosas, mis expectativas del trabajo, mis ganas de ver un cambio en todos. Se trata de interactuar juntos para alcanzar metas compartidas (Katz, 2005).

En las siguientes sesiones este sentimiento de ir más allá me llevó a enviar pequeñas tareas, las cuales -más que alcanzar productos- buscaron abrir espacios de comunicación y acercamiento a lo cotidiano del niño. Este pequeño paso nos abre, al padre y a mí, hacia el aprendizaje, hacia la comprensión del niño y su realidad, más que al desarrollo de habilidades, destrezas o conductas. Las tareas fueron pequeñas preguntas que buscaban respuestas tanto para el padre como para mí, cada semana enviaba unas cuantas y el padre debía traer las respuestas la próxima sesión. Las primeras tareas eran pequeñas preguntas que referían al conocimiento del niño, como por ejemplo: ¿qué le gustaba jugar?, ¿qué le gustaba comer?, ¿cuál era su juguete favorito?, ¿qué solía comer y qué no?, entre otros. Envíe tareas durante 7 sesiones, cada una con objetivos muy concretos que nos permitían una mejor comunicación y un mayor conocimiento mutuo.

Mi camino como docente hasta el momento estaba centrado en el padre, como actor principal, y no lograba verme, ni vernos los tres. Me cuestionaba ¿cómo se ve el padre?, ¿cómo se siente en su lugar de progenitor?, ¿cómo me ve en mi lugar de docente?, ¿cómo influyen estas sensaciones y percepciones en las actividades que hacemos con el niño? Quería ver, no a través de una pequeña ventana sino una más abierta, no cegarme por mis percepciones (Dobles et al., 1998). En mi papel como docente en el proceso de investigación se trata de comprender para acompañar.

Frente a esta reflexión emerge con fuerza ¿quién es el que tiene la discapacidad?, o ¿cuál es la discapacidad presente: la ceguera de lo cotidiano en las personas adultas o el autismo? Como personas adultas en ocasiones estamos atrapados en las concepciones de un paradigma tradicional y positivista, vemos la realidad como única, generalizable, objetiva, cuantificable. Desde una nueva mirada, ¿será el niño quien nos está mostrando uno de los caminos a seguir? 
Desde mi lugar de observadora participante evidencio la alegría, el entusiasmo y las ganas con que este niño trabaja junto con su padre con juegos como con el puré de papa y los colorantes, las texturas y la gelatina. Estos juegos eran espacios en los que el juego con las mezclas y diferentes texturas, un elemento muy importante a trabajar con las personas con autismo para ir logrando desensibilizarlos, era la excusa para jugar, para compartir, para experimentar con su padre. Como lo afirma Dolto (2000), el niño pequeño necesita esencialmente seguridad. La sola presencia del padre le brinda esta sensación. Lo tiene solo para él por 30 minutos dos veces a la semana, en las sesiones de trabajo conjunto. Parece poco tiempo, pero en realidad no se trata de la condición cronológica del tiempo sino de su sentido relativo.

Reitero que mi rol con el padre es fortalecerlo, apoyarlo y hacerle sentir que puede. Para la semana 7, en la actividad del juego con ubleck (una mezcla de maicena con agua) decido explicarle al padre el objetivo del juego antes de iniciar -el porqué se realizan actividades así con el niño y en qué le puede favorecer en el desarrollo-; esto le ayuda a participar con menos temor, más relajado. Compartir el saber es importante, pues con ello de lo que se trata es de horizontalizar las relaciones. Como apunta Freire (2006b), el educador progresista, capaz y serio, no solo debe enseñar muy bien su disciplina, sino desafiar al educando -en este caso al padre y la docente- a pensar críticamente en la realidad, se trata de darles herramientas para que lo hagan, siendo la información oportuna una de las más importantes. Con las "tareas" se buscaba eso: ver críticamente la realidad y buscar herramientas que nos ayudaran a todos para cambiar. En este momento se describen algunas de ellas con preguntas y respuestas del padre:

Escriba 10 palabras que describan a su hijo: selectivo, juguetón, capaz, cariñoso, tierno, lindo, independiente, concentrado, inteligente.

¿Con cuál de esas palabras se identifica más y por qué? Independiente, la forma de jugar, lo que le gusta es actuar solo, concentrado en lo que le gusta.

El padre responde las preguntas sin dar muchos detalles, aun así es un maravilloso inicio, en particular cuando analizo la palabra que utiliza para describir a su hijo y la que elige para representarlo. Menciona que el niño es independiente, efectivamente esta es una de las características del autismo, los niños se muestran generalmente autónomos y buscan realizar las tareas por sí solos, a su manera, y se alejan de los demás como lo menciona (Grandin y Scariano, 2011). El padre expresa en algunas de las tareas:

-"Esta fue una tarea grande... niña Rocío"

- Me hizo pensar con esta tarea... tuve que preguntar porque había cosas que no sabía, porque como solo lo tengo días, no sabía que comía fideos". 
doi: http://dx.doi.org/10.15359/ree.19-1.16

URL: http://www.una.ac.cr/educare

CORREO: educare@una.cr

La tarea se transforma en un buen instrumento para que el padre se exprese y me permite conocer sus creencias y percepciones. En esta sesión, conversar sin tener que limitarnos a preguntas de sí o no abre un espacio de comunicación nuevo. Es importante seguir con las tareas, poco a poco encausadas en oportunidades para que mire, escuche, atienda a su hijo, lo represente y signifique más allá de sus limitaciones. Confirmo que la estrategia de abrir un espacio particular para padre e hijo anudado por el juego es una herramienta acertada. Para Freire (2006b), la práctica educativa liberadora valora el ejercicio de la voluntad, la decisión, la resistencia, el papel de las emociones, los deseos, el papel de la conciencia de la propia historia. Buscaba sobre todo comprender ese papel de las emociones, el papel de la conciencia de la propia historia que esta familia y yo como docente íbamos a descifrar y que, poco a poco, comenzaba a pasar.

Trascurrieron sesiones de juego abordado de maneras diferentes: juegos con arena, texturas, pinturas entre otros, guiados por la docente con el padre y el niño. Juegos guiados solamente entre el padre y el niño con tareas especificas como de ensartes y demás, hasta que como docente me doy cuenta de que no tengo que guiar todo para que ocurran espacios grandiosos de juego libre y aprendizaje mutuo que me permite analizar todo lo que sucede desde otra perspectiva, para la auto reflexión.

\section{Principales hallazgos que nos llevaron a la transformación}

Es evidente el cambio en ambos, al principio el padre y yo nos mostrábamos muy estresados y centrados en la tarea, hasta que nuestro lenguaje, en la gestualidad descrita muestra como empiezan a cambiar. En las observaciones realizadas en la sesión 10 durante la actividad de juego libre el padre "espera por más tiempo para comprender lo que el niño trata de indicarle, lo mira, lo escucha, está más relajado" (18 de setiembre, 2012). Esta actitud resulta muy positiva para ambos.

Sin embargo, en realidad el amor que le tiene el padre a su hijo es lo que logra cambios de actitud, respuestas que antes no se veían, expresiones, sonrisas, alegría. Palabras del niño donde antes solo había silencio. Lo más importante en esta actividad "Juego con texturas" (juegos con diferentes texturas, con gel y colorantes) es lo que ese padre, el niño y yo hemos comenzado a percibir, los cambios de actitud. Como docente confirmé que no son las actividades ni los materiales los que hacen la diferencia sino la actitud construida desde la comprensión mutua, la aceptación, el intercambio.

Me pregunto si la actitud del niño se debe también a la actitud del padre. Un padre relajado implica un niño relajado. Durante el desarrollo de los encuentros, mi sensación es que estamos compartiendo un momento de alegría. Descubro las razones, muy concretas y simples, que solo he podido percibir después estas semanas: el niño ha ido cambiando sus actitudes por la disposición del padre al intercambio, al tiempo que el padre está dispuesto a compartir, y por esto es que el niño muestra una nueva actitud de alegría que compruebo cuando el niño 
viene más feliz los días de la semana en que su padre asiste a la clase. La relación entre ambos es distinta, amorosa, más flexible y relajada. El niño ha encontrado seguridad en su padre, "gracias a este sentimiento de seguridad, el niño puede construirse" (Dolto, 2000, p. 96). El padre que solo vive con el niño los fines de semana (porque se encuentra separado de la madre), le está apoyando, desde su manera, desde su posibilidad; el niño se da cuenta y acepta su amor.

Mi perspectiva como docente se amplía, ya no hay solo dos posibilidades, lo bueno y lo malo, sino particularidades, encuentros; potenciales que emergen de la historia de cada quien, del tiempo compartido, del gesto, la mirada, la sonrisa. Creo que lo más enriquecedor del proceso es transformarme, crecer personal y profesionalmente.

Al principio, mi intervención está centrada en la actividad, en las destrezas y habilidades del niño, en aquellas que según su diagnóstico no evolucionan como en los demás niños. Trabajo con la limitación y no con el potencial. Me preocupa que se cumpla la actividad, sin mirar nada más. A medida que pasan las semanas, me doy cuenta de que hay algo más que la actividad o el plan de sesión. Se trata de un trabajo entre humanos que no nos conocíamos aún, sin conocernos, como apunta Freire (2006b), no era posible llegar a la comprensión que libera.

Como docente debo tener claro mi papel. Como lección aprendida derivada de este proceso descubro que una importante parte de mi trabajo es el trabajo en equipo con la familia. Creo que el temor a los cambios, a lo incierto, nos produce un efecto de ceguera, que evidenciamos cerrándonos al trabajo con el otro (Maturana, 1996).

Todos estamos cambiando, el padre, el niño y yo. Me doy cuenta de que no soy la misma que inicia este proceso, la manera en la percepción de mi papel, del autismo, del rol de la familia ha cambiado, trascendiendo en sus implicaciones mi espacio laboral. Percibo el amor del padre hacia a su hijo y como este lo transforma. Como docente no tengo la verdad última, quiero que el padre encuentre en él mismo que puede hacer mucho más de lo que se imagina y que él tiene muchas habilidades, de esto estoy segura ahora. Quizá el reconocimiento de su potencial por mi parte, le ayude a construir esta idea.

Ningún proceso educativo está completo sin el docente, es cierto; pero no es suficiente su rol, en tanto su acción está íntimamente articulada con el papel desplegado por los niños y en este caso particular por el padre. Al creerme en cierto momento indispensable en el proceso, mi mirada excluyó a los otros. He estado en las sesiones anteriores, considerando el papel de los otros sin incluirme, la reflexión progresiva acerca de mi accionar me trae hasta este punto. Me libero del papel de juez que observa al padre, analiza sus características y sus conductas como independientes de sí. Me coloco en el lugar de docente reflexiva que se analiza en la complejidad de su papel, como persona en la limitación de su historia, como estudiante siempre en aprehendiencia, aceptando mi integralidad y la de los demás. Como señala Morin (2003), el conocimiento que proponemos es complejo porque: 
doi: http://dx.doi.org/10.15359/ree.19-1.16

URL: http://www.una.ac.cr/educare

CORREO: educare@una.cr

- reconoce que el sujeto humano que estudia está incluido en su objeto,

- concibe inseparablemente unidad y diversidad humanas,

- consideratodaslasdimensionesoaspectos, actualmentedisjuntosycompartimentalizados, de la realidad humana, que son físicos, biológicos, psicológicos, sociales, mitológicos, económicos, sociológicos, históricos, ...

- le encuentra un sentido a las palabras pedidas y despreciadas por las ciencias, incluidas las cognitivas: alma, mente, pensamiento (pp. 17-18).

Debo admitir que es después de doce semanas que me doy cuenta de esta ruptura epistemológica. No es simple cambiar, sin embargo, resulta aún más complejo aceptar los hallazgos hacia los cuales ese cambio nos lleva. La ruptura de las preconcepciones necesita angustia, dolor, goce; todo como parte de la reconstrucción epistémica de nuestra realidad (Morin, 2003).

El reflexionar acerca de mi actuar ha sido lo más difícil de todo este trabajo, pues pensaba que lo que hacía era lo mejor. Mis propósitos pedagógicos cambian, no se trata de que el padre aprenda o fortalezca su vínculo con el niño, se trata de aceptarlo en su particularidad, diferente a lo que mis preconcepciones han definido. A veces no queremos soltar, porque creemos que lo sabemos todo o lo podemos todo y, por miedo o por inseguridad, la ceguera nos atrapa; no podemos ver y seguimos actuando de manera mecánica. El currículo oculto invade nuestro quehacer, nuestra historia personal (habilidades, conocimientos, saberes, experiencias) incide en nuestro diario vivir.

La reflexión y el análisis me permiten colocarme en el lugar de horizontalidad con el padre: yo creía saber más, comprender más, desde un sentido positivo, como maestra, por lo cual mi misión era ayudarles a ver lo que no veía en su hijo. Sin embargo, descubro que existen muchos tipos de ceguera ante lo cotidiano y lo relacional. Cegueras originadas en muchas causas, pero con la misma consecuencia: la exclusión de la incertidumbre y el abrazo de la certeza individual (Morin, 2003). Olvidamos que la riqueza se encuentra en la construcción conjunta, en la diversidad, en la aceptación y la comprensión de nuestras diferencias. Al igual que el padre yo estaba impidiendo ese libre desarrollo del niño y su familia, estaba dirigiendo el proceso, tomando como foco solo mi mirada de la realidad.

Las oportunidades -más que organización de espacios, tiempos o materiales- son apertura a las relaciones respetuosas de la singularidad, que se abren cuando estamos dispuestos a escuchar al otro, a no organizar su actuar; sino a responder y validar ese actuar, sin premura o expectativas unidireccionales (Núñez, 2010). Mi papel como docente es de apoyo, de estratega que facilita al niño y el padre un escenario diferente para sus interacciones, un escenario pedagógico donde el padre puede probar nuevas maneras de interconectarse con su 
hijo. La ausencia de certeza, que me angustia tanto en todo el proceso, es, sin embargo, la que potencia los cambios en nuestra realidad (Maturana, 1996).

Es igualmente importante en este giro de lugares considerar que no es el resultado de una variante, sino un conjunto complejo de relaciones y dimensiones que toca a todos los participantes. Como propone Latorre (2004), la investigación acción interpreta lo que ocurre desde el punto de vista de quienes actúan e interactúan en la situación problema. Estas transformaciones en cada uno buscan siempre un cambio en la colaboración, la democratización del proceso, en función de la comunicación.

Los cambios de cada uno de los participantes nos son superficiales, en particular el mío como docente, mis preocupaciones, mis dudas, las preguntas, la incertidumbre hacia lo que podía suceder o no, se caen muchas creencias o absolutos que a veces tenemos construidos o aprendidos. Son elementos de los que ahora estoy consciente y puedo analizar. Freire (2007) expresa que para cambiar el mundo no es solamente la educación, sino que esta cambia a las personas que pueden hacer una diferencia en este mundo.

Nunca vamos a mejorar o cambiar si no extendemos la mirada hacia nosotros mismos. Como dice Freire textualmente, "es pensando críticamente la práctica de hoy o la de ayer como puedo mejorar la próxima" (Freire, 2006, p. 40a).

El resultado de este proceso con el padre fue abrir una oportunidad para la mirada conjunta, para percibir los detalles que fortalecen nuestros vínculos, los momentos en que su hijo le pone atención, le observa con detenimiento, le escucha mientras él le habla y cómo se comunican.

El vínculo que han establecido, se amplía, sin barreras, sin palabras que hieren o se centran en lo que no puede, en lo que es diferente (Dolto, 1998). La caída de las barreras nos permite ver un padre feliz, demostrando afecto a su hijo, disfrutando con él, sin pensar o percibir a su hijo desde una etiqueta, "mi hijo es autista"me dice, "ahora sí puedo presentarlo a mis amigos". Esta nueva percepción del padre es otro de los logros más importantes del proceso. El amor de un padre o una madre es mucho más fuerte que cualquier otra cosa.

El tiempo nos enseña que no todo lo podemos ver al instante, necesitamos oportunidades, espacios, período para mirar con nuevas perspectivas las situaciones de la vida (Rojas, 2011). En ciertos momentos me preocupé por el tiempo, por las actividades, porque todo fuera lo mejor; pero a veces son los detalles y la paciencia los que nos enseñan, dejando ver el verdadero valor de los actos, en nuestro caso vivenciados en las diferentes sesiones realizadas conjuntamente.

Más allá del tiempo, el análisis nos permitió descubrir, a mí como docente, un padre orgulloso de su hijo, a un padre que se ríe con él, algo que tal vez no había notado en lo que llevábamos compartiendo. Al padre, le permite encontrar a su hijo más allá de una discapacidad y, al niño, le muestra dos personas adultas emocionadas e interesadas en su bienestar (Núñez, 2010). 
REVISTA EleCtrónica EdUCARE (EdUCARE EleCtronic JOURNAL) EISSN: 1409-4258 VOL. 19(1) ENERO-ABRIL, 2015: 297-309

doi: http://dx.doi.org/10.15359/ree.19-1.16

URL: http://www.una.ac.cr/educare

CORREO: educare@una.cr

La investigación acción nos permite reflexionar, devolvernos, analizar, pero sobre todo, como apunta Latorre (2004), ayuda a mejorar la práctica a través de su trasformación, al tiempo que procura comprenderla. En este caso comprender más a este padre que con cada tarea me hace transformarme en mi rol profesional, en mi práctica docente.

Cambiar es, entonces, una transición, un paso de un modo de ser, pensar o sentir a otro distinto, que en este proceso compete no solo al padre sino a todos los participantes, docente incluida. Una transición en mi manera de ver las cosas, en mi paradigma, en mi sentir y hacer (Rojas, 2011). Con el trabajo en conjunto vivimos una transición en un espacio y tiempo particulares, aunque durante el camino hubo momentos de tinieblas, debo admitir que son más las alegrías, aprendizajes y reflexiones.

Como apunta Dumas (citado por Tierno, 2005):"La vida es fascinante, solo hay que mirarla a través de las gafas correctas" (p. 219).

Comenzamos viendo las dificultades, lo negativo, el problema, la discapacidad; hoy vemos lo positivo, los potenciales, las oportunidades, las fortalezas que vienen aparejadas con lo negativo. El papel del docente debe ir orientado hacia la potenciación, la conciencia, la reflexión y la creación.

Como apunta Maturana (1996), "pienso que la tarea de la educación es crear un espacio de transformación en el convivir de las personas, para el vivir" ( $p$.140). Puedo concluir que es un proceso de aprendizaje y de resiliencia, que nos recuerda algo fundamental que a veces en el día a día olvidamos: la participación de la familia en los entornos educativos debe ser como protagonistas y no como receptores.

\section{Referencias}

Chaves, L. (2007). El paradigma cualitativo en la investigación acción: Una aproximación teórica. En L. Chaves, M. Díaz, J. García, G. Rojas y N. Solís (Eds.), Investigación-acción colaborativa: Un encuentro con el quehacer cotidiano del centro educativo para su transformación. San José, Costa Rica: INIE.

Dobles, M., Zúñiga, M. y García, J. (1998). Investigación en educación. Procesos, interacciones y construcciones. San José, Costa Rica: Editorial EUNED.

Dolto, F. (1998). Las etapas de la infancia: Nacimiento, alimentación, juego, escuela. Barcelona: Paidós.

Dolto, F. (2000). El niño y la familia. Barcelona: Paidós.

Ferrer, E. (2012). Exigir para educar (13a ed,). Madrid: Ediciones Palabra.

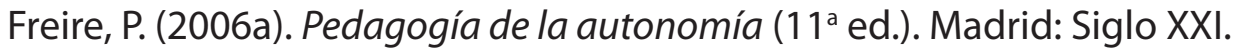


ReVISTA Electrónica EdUCARE (EdUCARE Electronic JOURNAL) EISSN: 1409-4258 VOL. 19(1) ENERO-ABRIL, 2015: 297-309

doi: http://dx.doi.org/10.15359/ree.19-1.16

URL: http://www.una.ac.cr/educare

CORREO: educare@una.cr

Freire, P. (2006b). Pedagogía de la indignación. Cartas pedagógicas en un mundo revuelto. ( $2^{\circ}$ ed.). Madrid: Ediciones Morata.

Freire, P. (2007). Cartas a quién pretende enseñar (4º reimp.). Buenos Aires: Siglo XXI.

Grandin, T. y Scariano, M. (2011). Atravesando las puertas del autismo: Una historia de esperanza y recuperación. Barcelona: Paidós.

Jara, Ó. (1994). Para sistematizar experiencias. Una propuesta teórico y práctica. San José, Costa Rica: Alforja.

Katz, L. (2005). Perspectivas educativas en la primera infancia. Santiago, Chile: Organización Mundial para la Educación Preescolar.

Kotliarenco, M. A., Cáceres, I. y Álvarez, C. (2011). La pobreza desde la mirada de la resiliencia. En M. A. Kotliarenco, I. Cáceres y C. Álvarez (Eds.), Resiliencia: Construyendo en adversidad (pp. 21-31). Santiago: CEANIM. Recuperado de http://www.resiliencia. cl/investig/Res-CAdversidad.pdf

Latorre, A. (2004). La investigación-acción: Conocer y cambiar la práctica. Barcelona: Graó.

Maturana, H. (1996). Transformación en la convivencia (2a ed.). Santiago: Dolmen Ediciones.

Morin, E. (2003). El Método V. La humanidad de la humanidad: La identidad humana. Madrid: Catedra.

Núñez, B. (2010). El niño discapacitado: Su familia y su docente. Buenos Aires: Grupo Editor Multimedial.

Rojas, E. (2011). No te rindas. Madrid: Grupo Planeta.

Tierno, B. (2005). Aprendiz de sabio. Barcelona: Editorial Grijalbo.

\section{Cómo citar este artículo en APA:}

Rodríguez-Roblero, M. (enero-abril, 2015). Trabajo conjunto familia y docente, el caso de un padre con su hijo autista: Una experiencia de investigación acción. Revista Electrónica Educare, 19(1), 297-309. doi: http://dx.doi.org/10.15359/ree.19-1.16

Nota: Para citar este artículo en otros sistemas puede consultar el hipervínculo "Como citar el artículo" en la barra derecha de nuestro sitio web:

http://www.revistas.una.ac.cr/index.php/EDUCARE/index 